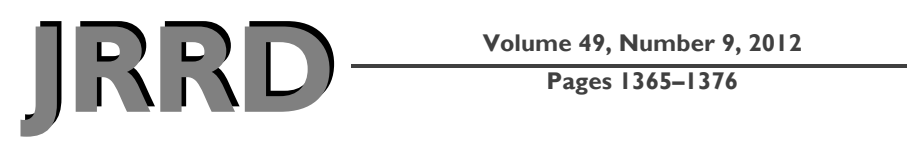

\title{
Regional cortical and trabecular bone loss after spinal cord injury
}

\author{
Shauna Dudley-Javoroski, PT, PhD; Richard K. Shields, PT, PhD, FAPTA* \\ Physical Therapy and Rehabilitation Science, Carver College of Medicine, The University of Iowa, Iowa City, IA
}

\begin{abstract}
Spinal cord injury (SCI) triggers rapid loss of trabecular bone mineral density (BMD) in bone epiphyses and a loss of cortical cross-sectional area (CSA) in bone diaphyses, increasing fracture risk for people with SCI. The purpose of this study was to measure trabecular BMD and cortical CSA loss at several previously unexamined lower-limb sites (4\% fibula, $12 \%$ femur, $86 \%$ tibia, cortical) in individuals with SCI. Using peripheral quantitative computed tomography, we scanned 13 participants with SCI longitudinally and 16 on one occasion; 21 participants without SCI served as controls. In the first year post-SCI, $15 \%$ to $35 \%$ of BMD was lost at the distal femur, proximal tibia, and distal fibula. Bone loss at the distal fibula accelerated between 1 and 2 years post-SCI. BMD at these sites reached a steady state value of $\sim 50 \%$ of the non-SCI value 4 years post-SCI. At the tibia diaphysis, cortical CSA decline was slower, eventually reaching $65 \%$ of the non-SCI value. Because of the extensive loss of bone observed at these sites, careful consideration needs to be given to the dose of musculoskeletal stress delivered during rehabilitation interventions like standing, muscle electrical stimulation, and aggressive stretching of spastic muscles.
\end{abstract}

Key words: adaptation, bone mineral density, cortical bone, fracture, neurological, osteoporosis, paralysis, rehabilitation, spinal cord injury, trabecular bone.

\section{INTRODUCTION}

New discoveries in bone biology emphasize that bone is a dynamic tissue that senses and adapts to a wide array of stimuli. The key to bone's adaptive capacity is its ability to detect, amplify, and transduce signals of various origins into cellular remodeling activities. Osteocyte cytoplasmic projections in the lacunocanalicular system sense interstitial fluid movement caused by mechanical loads [1]. Osteocytes communicate with one other via gap junctions [2] to transduce these small mechanical signals into molecular cascades that activate bone remodeling cells [3]. Bone can adapt to subtle, pervasive mechanical loads (vibration) [4] and to the largest episodic voluntary loads experienced by athletes [5-6]. This basic process is regulated by the endocrine system and may also bear upon an organism's future endocrine function, because osteocytes and adipose cells share a common marrow stem cell origin [7]. Hypothalamic nuclei directly modulate bone adaptation via the actions of leptin and other neural signaling molecules [8]. Therefore, far from being inert scaffolding, bone is an important endocrine organ that rehabilitation specialists strive to "regenerate" through timely prescribed doses of stress.

Spinal cord injury (SCI) presents a massive perturbation to all the systems that regulate bone health. The predictable result is derangement of bone homeostasis, culminating in loss of bone mineral density (BMD) in paralyzed limbs. BMD decline compromises bone

\footnotetext{
Abbreviations: $\mathrm{ANOVA}=$ analysis of variance, $\mathrm{BMD}=$ bone mineral density, CSA = cross-sectional area, DEXA = dual Xray absorptiometry, $\mathrm{pQCT}=$ peripheral quantitative computed tomography, SCI = spinal cord injury, SD = standard deviation. *Address all correspondence to Richard K. Shields, PT, PhD, FAPTA; Physical Therapy and Rehabilitation Science, The University of Iowa, 1-252 Medical Education Building, Iowa City, IA 52242; 319-335-9791; fax: 319-3359707. Email: richard-shields@uiowa.edu http://dx.doi.org/10.1682/JRRD.2011.12.0245
} 
strength, increasing the risk of fracture for people with SCI [9]. Fractures frequently occur during falls from a wheelchair, but even small external forces during transfers or range-of-motion exercises can be sufficient to yield a fracture [10-11]. When fractures occur in individuals with SCI, they often go unnoticed and are often accompanied by delayed diagnosis, hospitalization, and medical complications [11]. Although pharmacologic treatments to prevent BMD loss are under development [12], no drug-based method currently exists to prevent or treat post-SCI osteoporosis. Rehabilitation specialists have been on the forefront of prescribing nonpharmacologic bone-preservation interventions. Careful prescription of therapeutic stress requires that rehabilitation specialists understand the ideal "windows" for intervention and the bone tissue sites that are at greatest risk for BMD loss. Depending on the anatomic site, BMD decline may conclude by 4 to 7 yr post-SCI [13-14]. Depending on the dose of stress applied, rehabilitation interventions may be most efficacious before the newly adapted bone tissue reaches a low-BMD steady state [15-16].

Several gaps exist in our understanding of BMD loss after SCI. Information on bone loss within the first 6 mo post-SCI is limited, with estimates ranging from 2.0 to 13.5 percent loss for various lower-limb locations [1719]. The advent of peripheral quantitative computed tomography (pQCT), a three-dimensional measurement technique, has revealed that BMD loss differs at trabecular bone sites of the distal and proximal epiphyses [20] and that bone loss at diaphyseal sites occurs as a result of cortical wall thinning, not by decline of BMD $[13,20]$. The implications of this are clear for the rehabilitation specialist charged with recommending a dose of stress for these tissues: fracture risk may be higher for some sites than others. At two of the sites at greatest fracture risk, the proximal tibia and the supracondylar femur [11], post-SCI rate of BMD decline has never been examined. A few previous studies of the ultradistal femur suggest that bone loss near these regions may be rapid and extensive ( $>50 \%$ loss) [13]. Whether BMD loss varies as a function of time post-SCI has not been determined with pQCT. One study that used dual X-ray absorptiometry (DEXA), a two-dimensional technique, suggested that BMD loss was higher for the first year postinjury than for the second year [21].

The question of a BMD steady state after SCI remains contentious. A DEXA study of SCI/non-SCI monozygotic twins suggested that BMD decline contin- ued throughout the life span and did not reach a steady state [22]. Other longitudinal work with pQCT found only small, nonsignificant BMD losses after 30 mo of follow-up, suggesting that a steady state had indeed developed [14]. The issue of whether BMD declines indefinitely after SCI bears upon the haste with which rehabilitation interventions may need to be implemented. Studies of electrically stimulated cycling suggest that BMD gains after chronic SCI are likely to be small [2324] and not universally beneficial to all anatomic sites [23]. Interventions that begin quickly after SCI have shown better efficacy [15,25-26]. Understanding each site's rate of BMD loss and steady state timing will help rehabilitation specialists more effectively educate their clients about post-SCI BMD loss.

The purpose of this study was to measure BMD loss at several previously unexamined (4\% from distal fibula, $12 \%$ from distal femur, $86 \%$ from distal tibia) lower-limb sites in people with SCI. We determined whether the rate of BMD loss differed as a function of time post-SCI and whether or not BMD reached a steady state value. We anticipated that the severity of BMD loss would vary among the anatomic regions examined. Specifically, we predicted that the normal loading differences between the tibia and fibula may have caused divergent adaptations to emerge in these bone sites.

\section{METHODS}

\section{Participants}

Twenty-nine individuals with complete SCI (American Spinal Injury Association Impairment Scale grade A) [27] participated in this study. An additional 21 individuals without SCI served as a control cohort. Demographic data appear in the Table. Exclusion criteria were a history of bone pathology (bone-specific metabolic disease, cancer, etc.), thyroid disorder, previous fracture at the scan sites, pregnancy, and medications known to affect bone metabolism. Participants with SCI were not excluded if they previously performed unilateral electrical stimulation training in our research [15]; however, previously trained limbs were excluded from this analysis.

Control individuals underwent a single bilateral assessment with pQCT. Participants with SCI underwent between 1 and 6 bilateral pQCT scans over the course of 1-5 yr. Bilateral values were averaged across limbs for each participant. 
Table.

Subject demographics.

\begin{tabular}{|c|c|c|c|c|c|}
\hline Participant & Sex & SCI LeveI & Age & SCI Duration (yr) & Time Bin \\
\hline \multicolumn{6}{|l|}{ SCI } \\
\hline 2 & M & T8 & 20 & 0.22 & $1,2,3,5$ \\
\hline 4 & M & C6 & 49 & 0.31 & 1,4 \\
\hline 5 & $\mathrm{~F}$ & T6 & 18 & 0.41 & 1,4 \\
\hline 6 & M & $\mathrm{T} 8$ & 19 & 0.41 & 1 \\
\hline 8 & M & $\mathrm{T} 8$ & 43 & 0.33 & $1,2,3$ \\
\hline 9 & M & T12 & 20 & 0.42 & 1,2 \\
\hline 10 & M & $\mathrm{T} 4$ & 16 & 0.15 & 1 \\
\hline 11 & M & C7-8 & 20 & 0.42 & 1 \\
\hline 12 & M & T8 & 72 & 0.11 & 1 \\
\hline 13 & M & T11 & 22 & 0.81 & 2,3 \\
\hline 18 & $\mathrm{~F}$ & C5-6 & 26 & 1.07 & 3 \\
\hline 19 & M & T6 & 34 & 1.77 & 3 \\
\hline 20 & M & T10 & 64 & 1.49 & 3 \\
\hline 21 & M & $\mathrm{T} 4$ & 24 & 3.41 & 4,5 \\
\hline 22 & $\mathrm{M}$ & T6 & 28 & 2.05 & 4 \\
\hline 23 & M & $\mathrm{T} 4$ & 26 & 4.56 & 5 \\
\hline 24 & M & T6 & 36 & 7.74 & 5 \\
\hline 25 & $\mathrm{M}$ & $\mathrm{T} 1$ & 49 & 24.23 & 5 \\
\hline 26 & $\mathrm{M}$ & $\mathrm{T} 4$ & 46 & 9.21 & 5 \\
\hline 27 & $\mathrm{~F}$ & T6 & 44 & 23.04 & 5 \\
\hline 35 & M & - & 50 & - & - \\
\hline 36 & M & - & 62 & - & - \\
\hline 37 & M & - & 24 & - & - \\
\hline 38 & M & - & 24 & - & - \\
\hline 39 & $\mathrm{~F}$ & - & 48 & - & - \\
\hline 40 & M & - & 61 & - & - \\
\hline 41 & M & - & 27 & - & - \\
\hline 42 & $\mathrm{~F}$ & - & 31 & - & - \\
\hline 43 & M & - & 30 & - & - \\
\hline 44 & M & - & 42 & - & - \\
\hline 45 & M & - & 23 & - & - \\
\hline 46 & M & - & 24 & - & - \\
\hline 47 & M & - & 50 & - & - \\
\hline 48 & M & - & 28 & - & - \\
\hline 49 & M & - & 24 & - & - \\
\hline 50 & $\mathrm{~F}$ & - & 22 & - & - \\
\hline
\end{tabular}


This study was conducted as a mixed cross-sectional and longitudinal design. SCI individuals 1-12 (Table) were first assessed soon after SCI $(<6 \mathrm{mo})$. The remaining participants enrolled at later post-SCI times. Thirteen individuals in the SCI cohort underwent repeat pQCT evaluation at intervals spanning at least 3 mo. To facilitate statistical analysis, we partitioned the data set into five time bins based on time post-SCI: $0-0.5 \mathrm{yr},>0.5-1 \mathrm{yr}$, $>1-2 \mathrm{yr},>2-4 \mathrm{yr}$, and $>4$ yr. If an individual was scanned on more than one occasion in a time bin, the mean of the scans was used.

\section{Peripheral Quantitative Computed Tomography Scan Procedure}

pQCT measurements were performed with a Stratec XCT 3000 densitometer (Stratec Medical; Pforzheim, Germany). Accuracy of this device is 2 percent (to the COMAC phantom); precision is $\pm 3 \mathrm{mg} / \mathrm{cm}^{3}$ for trabecular bone and $\pm 9 \mathrm{mg} / \mathrm{cm}^{3}$ for cortical bone [28]. This device is calibrated with respect to fat (fat density $=0 \mathrm{mg} / \mathrm{cm}^{3}$ ). Voxel size was $0.4 \mathrm{~mm}^{3}$, scanner speed was $25 \mathrm{~mm} / \mathrm{s}$, and slice thickness was $2.2 \mathrm{~mm}$.

pQCT images were obtained at set percentages of limb length. Using a tape measure, we measured tibia length between the most distal palpable tip of the medial malleolus and the most proximal edge of the medial tibial plateau. Femur length was measured between the most proximal palpable limit of the greater trochanter and the most distal limit of the lateral femoral condyle. An investigator passed the limb through the pQCT gantry and secured the participant's foot onto a footplate. Using an inclinometer placed just distal to the tibial tuberosity, the investigator adjusted the vertical height of the footplate in order to bring the pitch of the shank to horizontal. A radiology technician performed a scout view of the talocrural joint and placed a reference line at the distal tibial plateau, bisecting the region of highest density at the lateral side of the distal tibia. Using this reference line, the scanner obtained an image at 4 percent and at 66 percent of tibia length (measured from the distal end). The 4 percent site has been widely studied [13,19,29-31] and provides a window into degradation of the tibia trabecular lattice with SCI. The 66 percent location lacks a trabecular lattice and has previously been used to examine endocortical absorption of the tibia cortical shell, as estimated by cortical cross-sectional area (CSA) $[23,32]$. In addition to tibia BMD and CSA, we were interested in examining fibula adaptations to paralysis. The fibula serves as a muscle attachment site but experiences far lower body weight forces than the tibia [33]. We suspected that the functional differences between the tibia and fibula might cause divergent adaptations to emerge in neighboring bone sites. We therefore analyzed BMD of the fibula in the 4 percent tibia images.

Limitations of gantry movement required the participant to be repositioned for scans of the proximal tibia and distal femur. Several individuals lacked sufficient trunk mobility or hip abduction range to allow appropriate positioning for femur scans. For the entire data set (52 scan sessions), 11 femur scans could not be obtained. For the proximal tibia and femur scans, a radiology technician performed a scout view of the tibiofemoral joint and placed a reference line at the proximal limit of the tibia plateau. Using this reference line, the scanner obtained an image at 86 percent of tibia length (measured from the distal end). We scanned this location because the tibial tuberosity is visible at this site. We suspected that the loss of quadriceps contractions after paralysis could exacerbate bone loss at the insertion site of the patellar tendon. No previous studies have reported longitudinal BMD change of the proximal tibia. Finally, the radiology technician placed a reference line at the distal limit of the lateral femoral condyle. Using this reference line, the scanner obtained an image at 12 percent of femur length. We scanned this location rather than the 4 percent femur site [13] because the supracondylar region has a higher risk for fracture [11].

\section{Analysis Procedures}

To analyze "trabecular" sites (fibula, distal tibia, proximal tibia, distal femur), an investigator defined a region of interest that incorporated a small amount of soft tissue outside the periosteal margin. A threshold algorithm removed voxels below $200 \mathrm{mg} / \mathrm{cm}^{3}$, starting from the outer edge of the region of interest and moving inward. This removed all voxels corresponding to muscle and fat and defined the periosteal edge. Inside the periosteal border, densities higher than $400 \mathrm{mg} / \mathrm{cm}^{3}$ were defined as cortical/subcortical bone and values lower than this threshold were defined as trabecular bone. Cortical/subcortical voxels were excluded from further analysis. A $3 \times 3$ voxel filter then proofed the remaining image to detect pockets of high-density values. Voxels that had substantially higher BMD than the neighboring voxels were reassigned as subcortical bone and were excluded from further analysis. Trabecular BMD was 
obtained from the resulting image. Because the cortical shell is very thin at these anatomic sites (and is therefore subject to the partial-volume effect [34]), we report only trabecular BMD.

To analyze the "cortical" site (tibia diaphysis), an investigator defined a region of interest that incorporated a small amount of soft tissue outside of the periosteal margin. A threshold algorithm removed voxels below $280 \mathrm{mg} / \mathrm{cm}^{3}$, corresponding to muscle and fat. In the remaining area, densities higher than $710 \mathrm{mg} / \mathrm{cm}^{3}$ were defined as cortical bone and values lower than this threshold were defined as subcortical/trabecular bone. Subcortical and trabecular voxels were excluded from further analysis. Previous studies demonstrated that cortical BMD does not decline after SCI [20]. Instead, endocortical absorption gradually leads to thinning of the cortical wall, adversely affecting bone strength [29]. Thus, at the 66 percent tibia site, we report the area of the cortical wall in millimeters squared.

\section{Data Processing}

Mean \pm standard deviation (SD) BMD or CSA values were computed for each scanned site at each time bin. To examine the different rates of bone loss among the sampled sites, we obtained the difference in BMD or CSA between the non-SCI value and the bin 1 SCI value. This difference, expressed as a percentage of the non-SCI value, was divided by the mean time post-SCI for the scans contained in bin 1 (0.32 yr). This yielded a rate of $\mathrm{BMD}$ or CSA loss expressed in terms of percentage of non-SCI BMD or CSA lost per year of SCI. We continued by obtaining difference values among successive bins and computing similar rates of decline. For participants with long-duration SCI, we were interested in determining whether BMD and CSA remained steady in bin 5 ( $>4 \mathrm{yr}$ ) or declines continued at a low rate. We divided the nine individuals with bin 5 data into subcohorts based on time post-SCI. Five individuals with SCI duration $<10$ yr (mean $6.05 \mathrm{yr}$ ) were assigned to bin "5a," and four individuals with SCI >10 yr (mean $22.44 \mathrm{yr}$ ) were assigned to bin " $5 \mathrm{~b}$."

\section{Statistical Analysis}

To examine BMD and CSA adaptations across time post-SCI, we analyzed data for each scan site by using a one-way analysis of variance (ANOVA) followed by pairwise multiple comparisons (Tukey). To compare the rate of decline across time post-SCI, we examined percent decline from the non-SCI value across time. For each time bin, we used a one-way repeated measures ANOVA and pairwise multiple comparisons (Tukey) to test for differences in rate of bone loss between scan sites but within time bins. Significance was set to $\alpha<0.05$.

\section{RESULTS}

Figure 1 shows representative examples of pQCT images for SCI and non-SCI participants at all scan sites. For the non-SCI group, mean \pm SD BMD for the fibula, distal tibia, proximal tibia, and distal femur was $255.4 \pm$ 35.5, $272.1 \pm 28.3,175.2 \pm 30.1$, and $211.1 \pm 18.7 \mathrm{mg} / \mathrm{cm}^{3}$, respectively. CSA at the tibia diaphysis was $430.8 \pm$

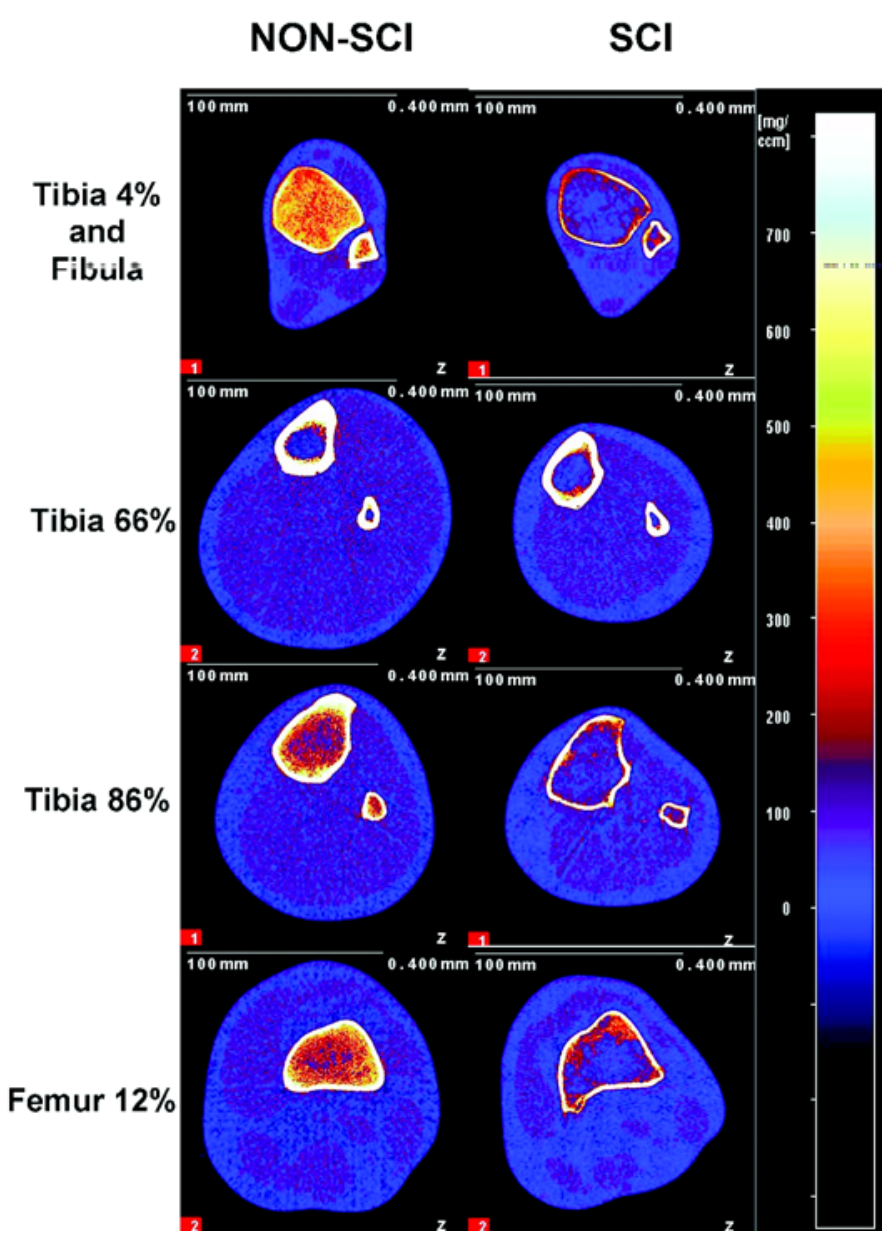

Figure 1.

Representative peripheral quantitative computed tomography images for participant without spinal cord injury $(\mathrm{SCl})$ and participant with SCl (3.67 yr). 
$53.5 \mathrm{~mm}^{2}$. BMD and CSA values for the SCI cohort across the five time bins appear in Figure 2. In this figure, non-SCI data appear as "bin 0." SCI BMD and CSA dropped significantly below the non-SCI value by bin 3 $(>1-2$ yr) for all sites except the fibula, which differed from non-SCI BMD from bin 4 onward $(p<0.001)$. Although several individuals showed a reduction in BMD and CSA in the first year, it did not reach significance for the average cohort (all $p>0.09$ ).

For all trabecular sites, BMD declined gradually until bin 4 ( $>2-4$ yr post-SCI: $p<0.001$ ). The tibia diaphysis, the cortical site, demonstrated a notably different pattern of decline; no significant differences emerged among bins 1-4 ( $p>0.17)$. Instead, CSA for bin 5 was significantly lower than all preceding post-SCI times $(p<0.001)$.
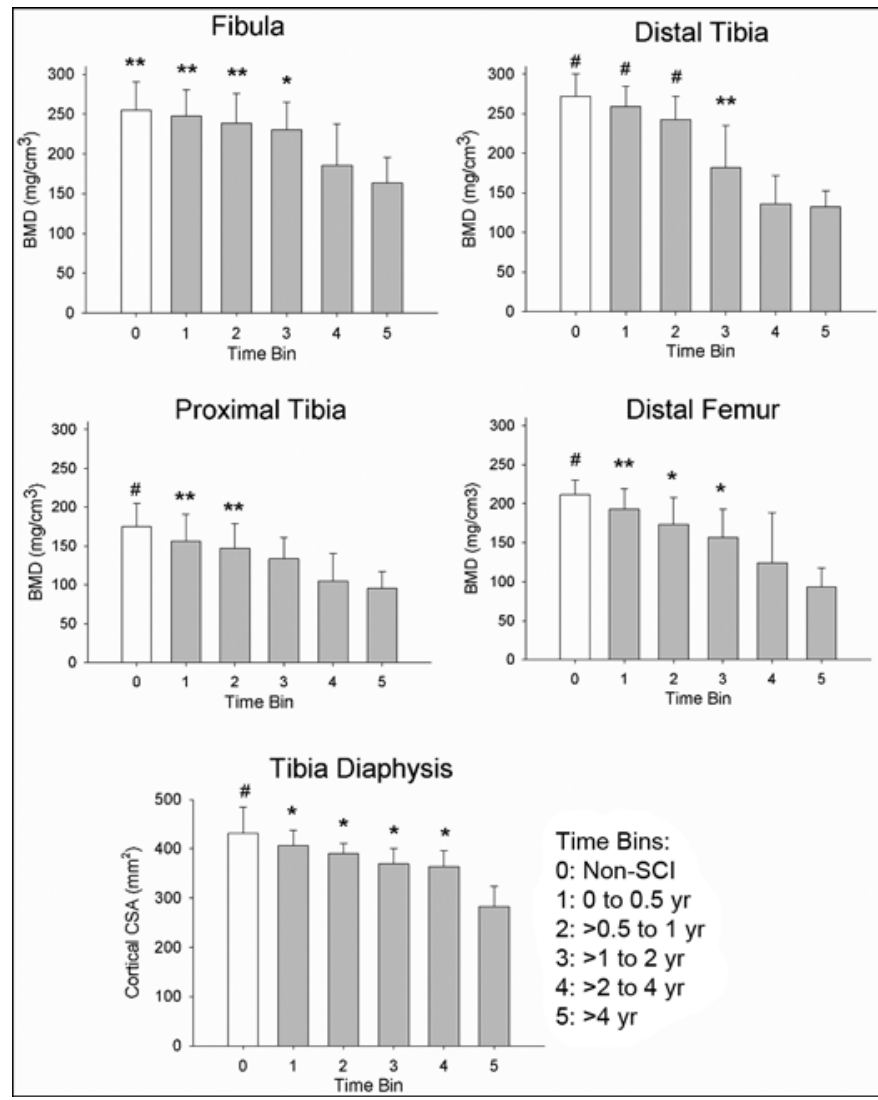

Figure 2.

Mean \pm standard deviation trabecular bone mineral density (BMD) or cortical cross-sectional area (CSA) for each sampled site across time. ${ }^{*}=$ Significantly different from bin $5,{ }^{* *}=$ significantly different from bins 4 and 5 , and \# = significantly different from bins $3-5$. All $p<0.05$. SCl $=$ spinal cord injury.
Figure 3 depicts SCI BMD and CSA values normalized to non-SCI values, arranged to compare differences among the sampled sites over time. No differences among sites in normalized BMD or CSA appeared in the first two time bins ( $<1 \mathrm{yr})(p>0.18)$. In bin $3(1-2 \mathrm{yr})$, normalized values for the fibula and the tibia diaphysis were greater than for the distal tibia $(p<0.01)$. These sites continued to differ from the distal tibia in bins 4 and 5 ( $p<$ 0.02 ). In bins 4 and 5 , the tibia diaphysis differed significantly from the proximal tibia and the distal femur $(p<$ 0.01 ). At the final time bin ( $>4$ yr post-SCI), normalized BMD was lowest for the distal femur, just 44.13 percent of the non-SCI BMD value. BMD was also very low for the distal tibia, at 48.67 percent of the non-SCI value.

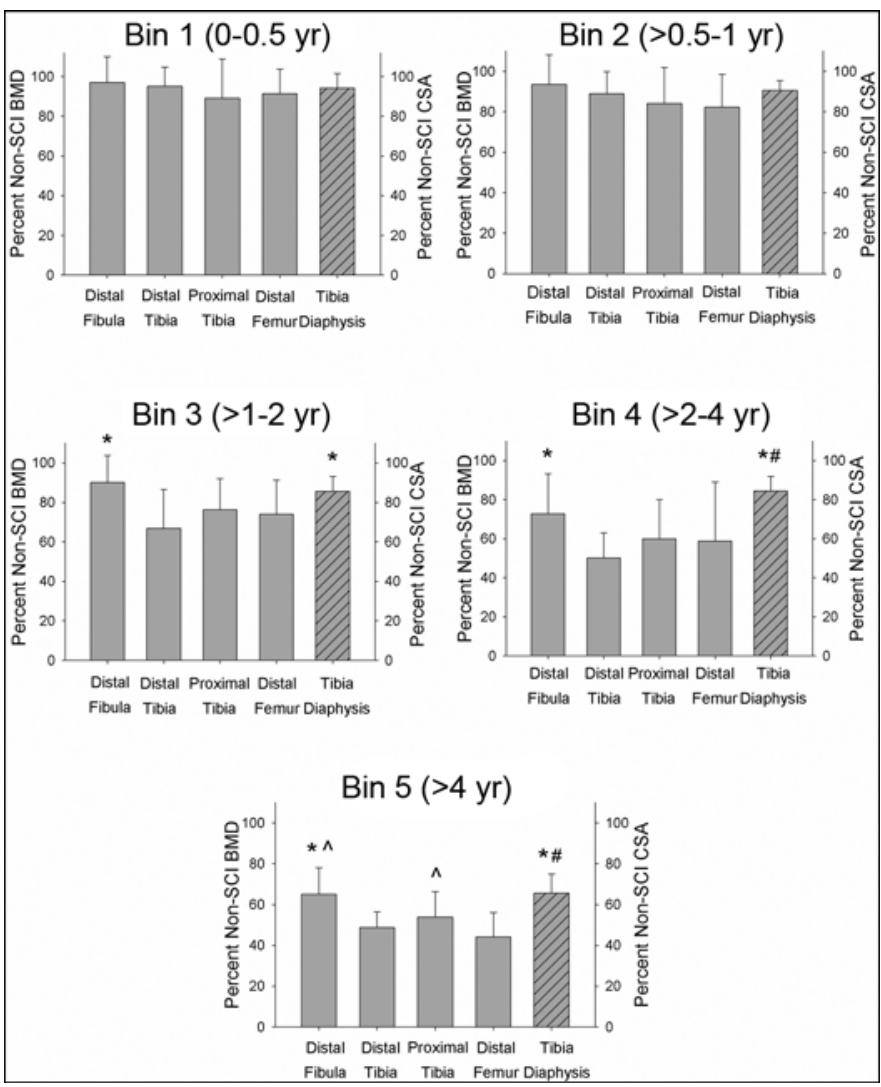

Figure 3.

Mean \pm standard deviation trabecular bone mineral density (BMD) or cortical cross-sectional area (CSA), normalized to nonspinal cord injury $(\mathrm{SCl})$ values. ${ }^{*}=$ Significantly different from distal tibia site, \# = significantly different from proximal tibia and distal femur sites, and ${ }^{\wedge}=$ significantly different from distal femur site. All $p<0.05$. Gray $=$ trabecular site, hatched $=$ cortical site . 
Even the site with the least bone loss, the tibia diaphysis, retained just 65.6 percent of the non-SCI value.

Figure 4(a) and 4(b) depicts the descriptive normalized rates of BMD and CSA decline for participants across time (note that bin 5 was partitioned into two subbins for this analysis). For the proximal tibia, tibia diaphyis, and distal femur, the rate of bone loss was highest in the first year postinjury and then declined to less than 12 percent loss per year thereafter. The distal tibia dem-

(a)

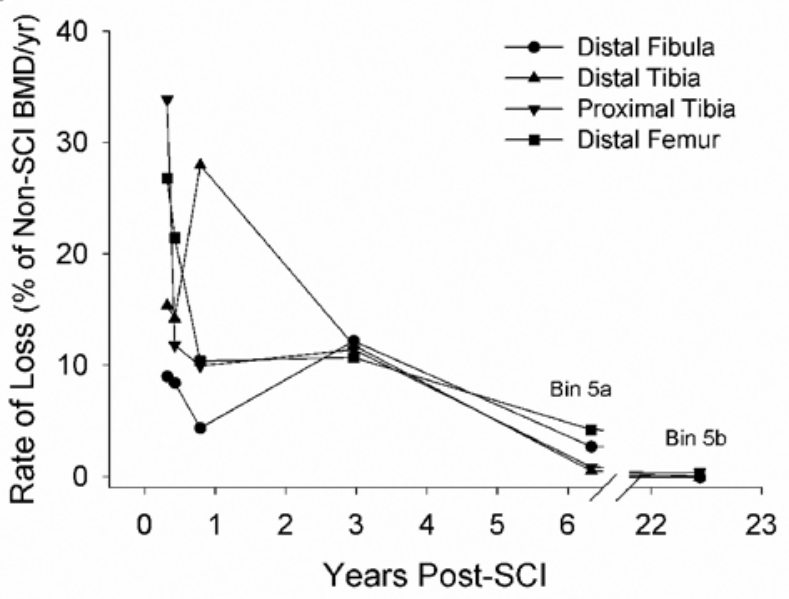

(b)

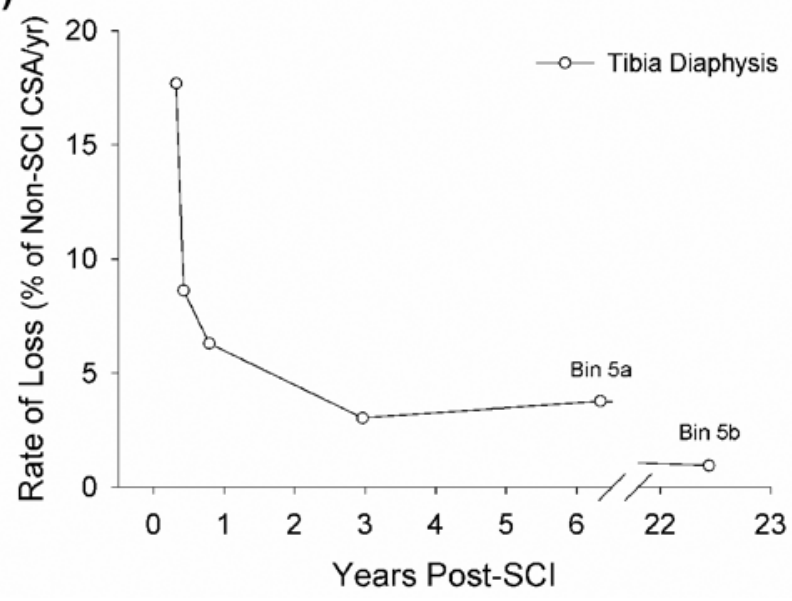

Figure 4.

Rate of bone loss as function of time after spinal cord injury (SCl). (a) Bone mineral density (BMD) loss for trabecular sites. (b) Cortical bone cross-sectional area (CSA) loss for tibia diaphysis. Data for time bin 5 were subdivided into bins $5 \mathrm{a}$ and $5 \mathrm{~b}$ to explore whether bone loss reached steady state ( $0 \%$ loss per year) in participants with very long duration $\mathrm{SCl}$ (>10 yr). onstrated a notably different pattern of decline than the other sampled sites, peaking instead between 1 and $2 \mathrm{yr}$ post-SCI (bin 3). BMD and CSA difference values between sub-bins $5 \mathrm{a}$ and $5 \mathrm{~b}$ were small, yielding a final rate of decline of $<1$ percent per year for all sites.

\section{DISCUSSION}

The purpose of this study was to measure BMD and CSA loss at several previously unexamined lower-limb sites in people with SCI. Specifically, there are no reports of BMD or CSA of the fibula, femur (12\%), and tibia (86\%) sites in this population. We investigated whether the rate of BMD and CSA loss differed as a function of time post-SCI and whether or not BMD and CSA reached a steady state value. Based on previous studies, we expected that the severity of bone loss would differ among the sampled sites. We anticipate that this information may be of particular interest to rehabilitation specialists who routinely need to stress the limbs of clients at various times after SCI.

\section{Timing of Bone Mineral Density and Cross-Sectional Area Loss}

Bone-sparing interventions that mechanically load the limbs [15,25-26] capitalize on the fact that immediately after SCI, bone strength for paralyzed clients should be similar to standard non-SCI values. As time post-SCI progresses and bone loss ensues, bones may become less able to withstand high loads, raising additional safety concerns for mechanical loading interventions. The timing of bone loss is a critical piece of information for the design and execution of such interventions. The only previous study of acute post-SCI bone loss indicated that distal tibia BMD declined by 5 percent in 6 mo $(p>0.05)$ and by 15 percent within 12 mo $(p<0.05)$ [19]. Distal tibia BMD decline in the present study was comparable (5\% in 6 mo, 11\% in 12 mo; Figure 3). Similar early BMD losses appeared at the proximal tibia and distal femur, and all three sites differed significantly from nonSCI BMD values by bin 3 ( $>1-2$ yr) (Figure 2). Although these findings suggest that in the first year post-SCI BMD did not differ significantly from non-SCI values, mechanical loading interventions that reintroduce physiologic forms of load at these skeletal sites may possess a reasonable degree of safety. However, there were insufficient subjects to clearly test statistically for a 
longitudinal effect within the first year, even when time was used as a covariate. Importantly, single-subject longitudinal data supports that large changes occur over the first 6 mo after injury. Given the multifactorial contributions to osteoporosis, extensive interparticipant variation within the first year is not surprising. Beyond $1 \mathrm{yr}$, bone losses at these sites reach significance and safety factors are now even more important when developing a therapeutic dose of stress as part of an exercise prescription.

In the latest time bins examined, steady state trabecular BMD appeared after 4 yr post-SCI; no trabecular site demonstrated a difference between bins 4 and 5 (Figure 2). One previous study of monozygotic twins suggested that BMD continues to decline throughout the life span postSCI [22]. That study employed DEXA, which cannot differentiate between cortical and trabecular bone loss. In the present study, we observed that tibia diaphysis cortical CSA differed significantly between bins 4 and 5, supporting that a steady state had not occurred. Moreover, we determined that cortical CSA continued to decline even in the individuals with the longest duration of injury (sub-bins $5 a$ and $5 b, p=0.002$ ). In contrast, no late trabecular BMD declines appeared in this time frame $(p>$ 0.05 ). Thus, the lack of a steady state in the aforementioned DEXA study may indicate that their BMD values were influenced by the continued decline of cortical bone superimposed over steady trabecular BMD in the DEXA image. Several more recent pQCT studies support that trabecular BMD does indeed reach a steady state between 4 to 7 yr post-SCI, depending on the anatomic site [1314]. These studies have not scrutinized cortical CSA at the tibia 66 percent site. However, 21 individuals with chronic SCI (>7.6 yr) followed longitudinally over $30 \mathrm{mo}$ showed no significant cortical CSA decline at 38 percent of tibia length, another diaphyseal site [14]. Our observation of prolonged CSA decline may indicate differences in bone loss between these two regions of the tibia diaphyis or may arise from the cross-sectional, not longitudinal, nature of our comparison. We believe it remains likely that tibia diaphyseal CSA does indeed reach a steady state value in chronic SCI. Additional longitudinal studies are required to address this question.

\section{Rate of Bone Mineral Density and Cross-Sectional Area Loss}

Because pQCT assessment of bone loss is not standard care for patients with SCI, clinicians have little basis by which to estimate skeletal integrity in their clients.
The ability to predict BMD based on time post-SCI would be a first step toward this goal. (BMD predictions must necessarily be placed into clinical context by epidemiology studies that correlate BMD to actual fracture risk.) Two previous cross-sectional studies offered exponential decay equations that used time post-SCI to estimate BMD at the tibia and femur distal epiphyses $[13,35]$. Our results suggest that fluctuations in the rate of $\mathrm{BMD}$ decline at the distal tibia may undermine the predictive power of a simple linear decay equation for this site. Figure 4 shows that the rate of BMD loss was higher between bins 2 and 3 (mean time $1.54 \mathrm{yr}$ ) than at other times post-SCI. Presumably, this elevated rate may be a spurious artifact due to the partially cross-sectional nature of the data set. To investigate this possibility, we separately examined rate of BMD decline for the five participants who contributed data longitudinally to each of bins 1-3 (Table). Among this longitudinal subset, rate of BMD loss of the distal tibia was higher between bins 2 and $3(26.3 \%)$ than for the preceding time intervals $(19.7 \%, 18.4 \%)$, consistent with what was observed in the mixed cross-sectional and longitudinal cohort. Thus, the acceleration of BMD loss at the distal tibia in the second year post-SCI warrants further investigation, particularly with longitudinal data.

\section{Relative Bone Loss Among Skeletal Sites}

This study examined relative bone loss in several previously unexamined sites that are pertinent to rehabilitation. Previous reports examined a more distal femur site (4\% of femur length, the femoral condyles) and noted severe decline in trabecular BMD. We opted to sample the 12 percent site instead because the supracondylar region has been strongly implicated as an at-risk site for fracture [11,36]. In our participants with SCI $>4 \mathrm{yr}$, just 44 percent of non-SCI BMD remained at the distal femur. Another study of a slightly more proximal femur site (15\% of length) found less bone loss (34\% loss of BMD) [37]. However, in previous reports, the sample size was limited ( $n=6)$, two individuals had incomplete SCI, and at least one individual had not yet reached the elapsed time predicted for the femur to reach steady state (4-7 yr) [13-14]. We believe that the steady state value demonstrated by our cohort illustrates the magnitude of supracondylar BMD loss associated with SCI. Figure 1 underscores how extensive the loss of trabecular lattice can be at this site in chronic SCI. Rehabilitation specialists 
should be aware of the high risk for fracture at supracondylar sites and educate their clients accordingly.

No previous studies have examined the longitudinal decline of BMD at the proximal tibia, another chief site of post-SCI fracture [11]. One previous cross-sectional report suggested that 47 percent of non-SCI BMD may remain at this site post-SCI [37]. Our data indicate that while overall BMD loss is less severe than at the distal femur (Figure 3), just 54 percent of non-SCI BMD may remain when this site reaches steady state.

Steady state BMD was similarly low at the distal tibia (49\% of non-SCI BMD), yet another common site for post-SCI fracture [11]. BMD at this location had been reported to be even lower by a previous cross-sectional study (36.9\% of non-SCI BMD) [13]. While we believe this lower value is attributable to differences in analysis parameters (peel method) between our study and theirs [38], our findings are in general agreement.

Before 1 yr post-SCI, no significant differences emerged among the sampled sites in BMD or CSA loss within a fixed time bin (Figure 3). After this time, fibula BMD and tibia diaphysis CSA generally showed less severe declines than the three other sites. A recent report offers a possible explanation for the relative preservation of bone in these locations [20]. Osteoclast and osteoblast activity occur primarily at the surfaces of existing bone structures [39-40]. In bone epiphyses, every trabecular rod and plate is a potential site for bone remodeling. In bone diaphyses, only the endocortical surface provides surface area for bone remodeling. If bone loss after SCI is time limited, then the low surface area present for bone remodeling limits the total amount of bone that can be lost in diaphyseal regions [20]. Lesser bone loss in diaphyses than in epiphyses is a consistent feature in previous reports $[13,20,29]$.

The pattern of bone loss at the distal fibula, a site with abundant trabecular bone (Figure 1), is interesting to consider. No previous studies have examined BMD of the fibula despite the fact that tibia/fibula fractures routinely occur after SCI $[11,36]$. In the present study, an SCI versus non-SCI difference appeared later for the fibula (bin 4) than for the adjacent distal tibia (bin 3) (Figure 2). Likewise, overall BMD loss in the fibula was significantly lower than for the distal tibia $(p<0.001)$ (Figure 3). Prior to SCI, the fibula experiences minimal weight bearing, serving chiefly as a muscle attachment site [33]. After SCI, perhaps the fibula loses only one source of routine load, while the adjacent distal tibia loses two sources (body weight and muscle forces). On the other hand, perhaps BMD of the distal tibia is more adversely affected by marrow cavity venous stasis [41] or is differently regulated by neural factors [42], such as leptin signaling [43], sympathetic nerve activity [44], or neuropeptide expression [45]. Differential BMD loss in adjacent fibula and tibia segments may provide a useful model for future investigations of neuroskeletal interactions.

\section{CONCLUSIONS}

In the first year post-SCI, between 15 and 35 percent of BMD is lost in three of the sites at greatest risk for fracture: the distal femur, proximal tibia, and distal tibia. Bone loss at the distal tibia appears to accelerate between 1 and 2 yr post-SCI, compounding the difficulty of predicting BMD based on time postinjury. BMD at these sites eventually reaches a steady state sometime after $4 \mathrm{yr}$ post-SCI. At bone epiphyses, steady state BMD is only $\sim 50$ percent of non-SCI values. At the tibia diaphysis, bone loss through endocortical resorption is slower but may persist longer, eventually reaching 65 percent of the non-SCI CSA value. A limitation of this study was the number of subjects that had data points across all the time bins examined. Future studies designed to predict absolute change in BMS across time will require a more extensive data set.

Rehabilitation interventions often reintroduce mechanical loads to the limbs (or may incidentally load the limbs during the course of other activities). Careful consideration needs to be given to the ability of the skeletal system of paralyzed limbs to withstand these loads. Shear forces across the axis of osteoporotic bones should be minimized because of the risk of fracture [46-47]. Mechanical loading interventions that begin early after SCI $[15,26]$, when BMD is nearly normal, may offer greater safety and may be more efficacious than interventions applied after chronic SCI [16].

\section{ACKNOWLEDGMENTS}

\section{Author Contributions:}

Project development: S. Dudley-Javoroski, R. K. Shields. Data collection and analysis: S. Dudley-Javoroski, R. K. Shields. Writing of manuscript: S. Dudley-Javoroski, R. K. Shields. Obtained funding: R. K. Shields. 
Financial Disclosures: The authors have declared that no competing interests exist.

Funding/Support: This material was based on work supported in part by awards to R. K. Shields from the National Institutes of Health (grants R01NR010285 and R01HD062507), the Department of Veterans Affairs, the Christopher and Dana Reeve Foundation, and the Craig H. Neilsen Foundation.

Additional Contributions: The authors gratefully acknowledge the assistance of Deanna Frei, RT (R)(CT)(BD)(CBDT), and April Miller, RTR. The authors thank Daniel Schiferl and Christopher Gordon, $\mathrm{PhD}$, for pQCT technical assistance.

Institutional Review: The protocol was approved by the University of Iowa Human Subjects Office Institutional Review Board. All individuals provided written informed consent before participating.

Participant Follow-Up: The authors do not plan to inform participants of the publication of this study.

\section{REFERENCES}

1. Weinbaum S, Cowin SC, Zeng Y. A model for the excitation of osteocytes by mechanical loading-induced bone fluid shear stresses. J Biomech. 1994;27(3):339-60. [PMID:8051194] http://dx.doi.org/10.1016/0021-9290(94)90010-8

2. Taylor AF, Saunders MM, Shingle DL, Cimbala JM, Zhou Z, Donahue HJ. Mechanically stimulated osteocytes regulate osteoblastic activity via gap junctions. Am J Physiol Cell Physiol. 2007;292(1):C545-52. [PMID:16885390] http://dx.doi.org/10.1152/ajpcell.00611.2005

3. Ehrlich PJ, Lanyon LE. Mechanical strain and bone cell function: a review. Osteoporos Int. 2002;13(9):688-700. [PMID:12195532] http://dx.doi.org/10.1007/s001980200095

4. Rubin C, Turner AS, Mallinckrodt C, Jerome C, McLeod $\mathrm{K}$, Bain S. Mechanical strain, induced noninvasively in the high-frequency domain, is anabolic to cancellous bone, but not cortical bone. Bone. 2002;30(3):445-52.

[PMID:11882457] http://dx.doi.org/10.1016/S8756-3282(01)00689-5

5. Heinonen A, Sievänen H, Kannus P, Oja P, Vuori I. Sitespecific skeletal response to long-term weight training seems to be attributable to principal loading modality: a pQCT study of female weightlifters. Calcif Tissue Int. 2002;70(6):469-74. [PMID:12016461]

http://dx.doi.org/10.1007/s00223-001-1019-9

6. Heinonen A, Sievänen H, Kyröläinen H, Perttunen J, Kannus P. Mineral mass, size, and estimated mechanical strength of triple jumpers' lower limb. Bone. 2001;29(3):279-85.

[PMID:11557373] http://dx.doi.org/10.1016/S8756-3282(01)00574-9

7. Sarraf CE, Otto WR, Eastwood M. In vitro mesenchymal stem cell differentiation after mechanical stimulation. Cell
Prolif. 2011;44(1):99-108. [PMID:21199014]

http://dx.doi.org/10.1111/j.1365-2184.2010.00740.x

8. Takeda S, Elefteriou F, Levasseur R, Liu X, Zhao L, Parker KL, Armstrong D, Ducy P, Karsenty G. Leptin regulates bone formation via the sympathetic nervous system. Cell. 2002;111(3):305-17. [PMID:12419242] http://dx.doi.org/10.1016/S0092-8674(02)01049-8

9. Zehnder Y, Lüthi M, Michel D, Knecht H, Perrelet R, Neto I, Kraenzlin M, Zäch G, Lippuner K. Long-term changes in bone metabolism, bone mineral density, quantitative ultrasound parameters, and fracture incidence after spinal cord injury: a cross-sectional observational study in 100 paraplegic men. Osteoporos Int. 2004;15(3):180-89. [PMID:14722626] http://dx.doi.org/10.1007/s00198-003-1529-6

10. Keating JF, Kerr M, Delargy M. Minimal trauma causing fractures in patients with spinal cord injury. Disabil Rehabil. 1992;14(2):108-9. [PMID:1600181] http://dx.doi.org/10.3109/09638289209167081

11. Fattal C, Mariano-Goulart D, Thomas E, Rouays-Mabit H, Verollet C, Maimoun L. Osteoporosis in persons with spinal cord injury: the need for a targeted therapeutic education. Arch Phys Med Rehabil. 2011;92(1):59-67.

[PMID:21187206]

http://dx.doi.org/10.1016/j.apmr.2010.09.019

12. Bubbear JS, Gall A, Middleton FR, Ferguson-Pell M, Swaminathan R, Keen RW. Early treatment with zoledronic acid prevents bone loss at the hip following acute spinal cord injury. Osteoporos Int. 2011;22(1):271-79.

[PMID:20358358] http://dx.doi.org/10.1007/s00198-010-1221-6

13. Eser P, Frotzler A, Zehnder Y, Wick L, Knecht H, Denoth J, Schiessl H. Relationship between the duration of paralysis and bone structure: a pQCT study of spinal cord injured individuals. Bone. 2004;34(5):869-80. [PMID:15121019] http://dx.doi.org/10.1016/j.bone.2004.01.001

14. Frotzler A, Berger M, Knecht H, Eser P. Bone steady-state is established at reduced bone strength after spinal cord injury: a longitudinal study using peripheral quantitative computed tomography (pQCT). Bone. 2008;43(3):549-55. [PMID:18567554] http://dx.doi.org/10.1016/j.bone.2008.05.006

15. Shields RK, Dudley-Javoroski S. Musculoskeletal plasticity after acute spinal cord injury: effects of long-term neuromuscular electrical stimulation training. J Neurophysiol. 2006;95(4):2380-90. [PMID:16407424]

http://dx.doi.org/10.1152/jn.01181.2005

16. Shields RK, Dudley-Javoroski S. Musculoskeletal adaptation in chronic spinal cord injury: effects of long-term soleus electrical stimulation training. J Neurorehabil Neural Repair. 2006;21:169-79. http://dx.doi.org/10.1177/1545968306293447 
17. Clark JM, Jelbart M, Rischbieth H, Strayer J, Chatterton B, Schultz C, Marshall R. Physiological effects of lower extremity functional electrical stimulation in early spinal cord injury: lack of efficacy to prevent bone loss. Spinal Cord. 2007;45(1):78-85. [PMID:16636686]

http://dx.doi.org/10.1038/sj.sc.3101929

18. Lai CH, Chang WH, Chan WP, Peng CW, Shen LK, Chen JJ, Chen SC. Effects of functional electrical stimulation cycling exercise on bone mineral density loss in the early stages of spinal cord injury. J Rehabil Med. 2010;42(2):150-54. [PMID:20140411] http://dx.doi.org/10.2340/16501977-0499

19. Frey-Rindova P, de Bruin ED, Stüssi E, Dambacher MA, Dietz V. Bone mineral density in upper and lower extremities during 12 months after spinal cord injury measured by peripheral quantitative computed tomography. Spinal Cord. 2000;38(1):26-32. [PMID:10762194]

http://dx.doi.org/10.1038/sj.sc.3100905

20. Rittweger J, Goosey-Tolfrey VL, Cointry G, Ferretti JL. Structural analysis of the human tibia in men with spinal cord injury by tomographic (pQCT) serial scans. Bone. 2010;47(3):511-18. [PMID:20561989] http://dx.doi.org/10.1016/j.bone.2010.05.025

21. Alekna V, Tamulaitiene M, Sinevicius T, Juocevicius A. Effect of weight-bearing activities on bone mineral density in spinal cord injured patients during the period of the first two years. Spinal Cord. 2008;46(11):727-32.

[PMID:18443599]

http://dx.doi.org/10.1038/sc.2008.36

22. Bauman WA, Spungen AM, Wang J, Pierson RN Jr, Schwartz E. Continuous loss of bone during chronic immobilization: a monozygotic twin study. Osteoporos Int. 1999; 10(2):123-27. [PMID:10501792] http://dx.doi.org/10.1007/s001980050206

23. Frotzler A, Coupaud S, Perret C, Kakebeeke TH, Hunt KJ, Donaldson NN, Eser P. High-volume FES-cycling partially reverses bone loss in people with chronic spinal cord injury. Bone. 2008;43(1):169-76. [PMID:18440891] http://dx.doi.org/10.1016/j.bone.2008.03.004

24. Ashe MC, Eng JJ, Krassioukov AV, Warburton DE, Hung C, Tawashy A. Response to functional electrical stimulation cycling in women with spinal cord injuries using dualenergy X-ray absorptiometry and peripheral quantitative computed tomography: a case series. J Spinal Cord Med. 2010;33(1):68-72. [PMID:20397446]

25. Dudley-Javoroski S, Shields RK. Dose estimation and surveillance of mechanical loading interventions for bone loss after spinal cord injury. Phys Ther. 2008;88(3):387-96. [PMID:18202080] http://dx.doi.org/10.2522/ptj.20070224

26. Dudley-Javoroski S, Shields RK. Asymmetric bone adaptations to soleus mechanical loading after spinal cord injury.
J Musculoskelet Neuronal Interact. 2008;8(3):227-38. [PMID:18799855]

27. American Spinal Injury Association. International standards for neurological classification of SCI. Atlanta (GA): American Spinal Injury Association; 2002.

28. Norland Medical Systems I. XCT 2000 Technical reference. Technical information. White Plains (NY): Norland Medical Systems; 2000.

29. Dionyssiotis Y, Trovas G, Galanos A, Raptou P, Papaioannou N, Papagelopoulos P, Petropoulou K, Lyritis GP. Bone loss and mechanical properties of tibia in spinal cord injured men. J Musculoskelet Neuronal Interact. 2007;7(1): 62-68. [PMID:17396008]

30. Eser P, Frotzler A, Zehnder Y, Denoth J. Fracture threshold in the femur and tibia of people with spinal cord injury as determined by peripheral quantitative computed tomography. Arch Phys Med Rehabil. 2005;86(3):498-504. [PMID:15759235] http://dx.doi.org/10.1016/j.apmr.2004.09.006

31. Coupaud S, Jack LP, Hunt KJ, Allan DB. Muscle and bone adaptations after treadmill training in incomplete Spinal Cord Injury: a case study using peripheral Quantitative Computed Tomography. J Musculoskelet Neuronal Interact. 2009;9(4):288-97. [PMID:19949287]

32. Eser P, Frotzler A, Zehnder Y, Schiessl H, Denoth J. Assessment of anthropometric, systemic, and lifestyle factors influencing bone status in the legs of spinal cord injured individuals. Osteoporos Int. 2005;16(1):26-34.

[PMID:15138665]

http://dx.doi.org/10.1007/s00198-004-1638-x

33. Funk JR, Rudd RW, Kerrigan JR, Crandall JR. The line of action in the tibia during axial compression of the leg. J Biomech. 2007;40(10):2277-82. [PMID:17141787] http://dx.doi.org/10.1016/j.jbiomech.2006.10.012

34. Hangartner TN, Gilsanz V. Evaluation of cortical bone by computed tomography. J Bone Miner Res. 1996;11(10): 1518-25. [PMID:8889852] http://dx.doi.org/10.1002/jbmr.5650111019

35. Coupaud S, McLean AN, Allan DB. Role of peripheral quantitative computed tomography in identifying disuse osteoporosis in paraplegia. Skeletal Radiol. 2009;38(10): 989-95. [PMID:19277646] http://dx.doi.org/10.1007/s00256-009-0674-1

36. Morse LR, Battaglino RA, Stolzmann KL, Hallett LD, Waddimba A, Gagnon D, Lazzari AA, Garshick E. Osteoporotic fractures and hospitalization risk in chronic spinal cord injury. Osteoporos Int. 2009;20(3):385-92.

[PMID:18581033] http://dx.doi.org/10.1007/s00198-008-0671-6

37. Rittweger J, Gerrits K, Altenburg T, Reeves N, Maganaris $\mathrm{CN}$, de Haan A. Bone adaptation to altered loading after spinal cord injury: a study of bone and muscle strength. 
JRRD, Volume 49, Number 9, 2012

J Musculoskelet Neuronal Interact. 2006;6(3):269-76. [PMID:17142949]

38. Dudley-Javoroski S, Shields RK. Longitudinal changes in femur bone mineral density after spinal cord injury: effects of slice placement and peel method. Osteoporos Int. 2010;21(6):985-95. [PMID:19707702]

http://dx.doi.org/10.1007/s00198-009-1044-5

39. Parfitt AM. Targeted and nontargeted bone remodeling: relationship to basic multicellular unit origination and progression. Bone. 2002;30(1):5-7. [PMID:11792557] http://dx.doi.org/10.1016/S8756-3282(01)00642-1

40. Downey PA, Siegel MI. Bone biology and the clinical implications for osteoporosis. Phys Ther. 2006;86(1):77-91. [PMID:16386064]

41. Chantraine A, van Ouwenaller C, Hachen HJ, Schinas P. Intra-medullary pressure and intra-osseous phlebography in paraplegia. Paraplegia. 1979;17(4):391-99.

[PMID:534112] http://dx.doi.org/10.1038/sc.1979.75

42. Sample SJ, Behan M, Smith L, Oldenhoff WE, Markel MD, Kalscheur VL, Hao Z, Miletic V, Muir P. Functional adaptation to loading of a single bone is neuronally regulated and involves multiple bones. J Bone Miner Res. 2008;23(9):1372-81. [PMID:18410233] http://dx.doi.org/10.1359/jbmr.080407

43. Rosen CJ, Klibanski A. Bone, fat, and body composition: evolving concepts in the pathogenesis of osteoporosis. Am J Med. 2009;122(5):409-14. [PMID:19375545] http://dx.doi.org/10.1016/j.amjmed.2008.11.027

44. Hill EL, Turner R, Elde R. Effects of neonatal sympathectomy and capsaicin treatment on bone remodeling in rats.
Neuroscience. 1991;44(3):747-55. [PMID:1721689] http://dx.doi.org/10.1016/0306-4522(91)90094-5

45. Irie K, Hara-Irie F, Ozawa H, Yajima T. Calcitonin generelated peptide (CGRP)-containing nerve fibers in bone tissue and their involvement in bone remodeling. Microsc Res Tech. 2002;58(2):85-90. [PMID:12203707] http://dx.doi.org/10.1002/jemt.10122

46. Hartkopp A, Murphy RJ, Mohr T, Kjaer M, BieringSørensen F. Bone fracture during electrical stimulation of the quadriceps in a spinal cord injured subject. Arch Phys Med Rehabil. 1998;79(9):1133-36. [PMID:9749697] http://dx.doi.org/10.1016/S0003-9993(98)90184-8

47. Frey Law LA, Shields RK. Femoral loads during passive, active, and active-resistive stance after spinal cord injury: a mathematical model. Clin Biomech (Bristol, Avon). 2004; 19(3):313-21. [PMID:15003348] http://dx.doi.org/10.1016/j.clinbiomech.2003.12.005

Submitted for publication December 22, 2011. Accepted in revised form March 16, 2012.

This article and any supplementary material should be cited as follows:

Dudley-Javoroski S, Shields RK. Regional cortical and trabecular bone loss after spinal cord injury. J Rehabil Res Dev. 2012;49(9):1365-76. http://dx.doi.org/10.1682/JRRD.2011.12.0245

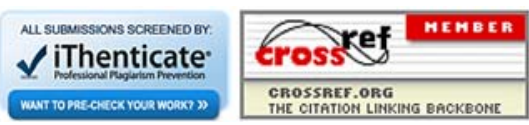

